\title{
An Integrated Expert System for Linear Scheduling Heavy Earthmoving Operations
}

\author{
Nizar Markiz and Ahmad Jrade \\ Department of Civil Engineering, University of Ottawa, Ottawa, ON, Canada K1N 6N5 \\ Correspondence should be addressed to Ahmad Jrade; ajrade@uottawa.ca \\ Received 25 April 2016; Accepted 16 June 2016 \\ Academic Editor: George Morcous
}

Copyright ( 2016 N. Markiz and A. Jrade. This is an open access article distributed under the Creative Commons Attribution License, which permits unrestricted use, distribution, and reproduction in any medium, provided the original work is properly cited.

\begin{abstract}
Heavy earthmoving operations are repetitive in nature and vulnerable to time-related restraints and uncertainties. Therefore, at the conceptual stage, scheduling these operations can take a linear form, known as linear schedule or line of balance (LOB). In such type of work, generating a preliminary line of balance for variable sequencing of activities is crucial. In this paper, an integrated expert system for determining preliminary linear schedules for heavy earthmoving operations at the conceptual stage is presented. The proposed system incorporates numerous factors that influence the analysis of earthmoving operations, which include geological and topographical parameters used to determine productivity rates at the conceptual stage. Also, the proposed system is capable of automatically generating a line of balance based on a stochastic scheduling technique via the metaheuristic simulated annealing intelligent approach to incorporate randomness and uncertainties in performing the associated activities. A parametric analysis is conducted in order to quantify the system's degree of accuracy. An actual case project is then utilized to illustrate its numerical capabilities. Generating accurate linear schedules for heavy earthmoving operations at the conceptual design stage is anticipated to be of major significance to infrastructure project stakeholders, engineers, and construction managers by detecting schedule's conflicts early in order to enhance overall operational logistics.
\end{abstract}

\section{Introduction}

Heavy earthmoving operations are repetitive by nature and vulnerable to diverse restraints and uncertainties. In order to ensure proper and continuous work flow, effective scheduling of earthmoving operations based on their productivity rates is crucial. Essentially, the performance of heavy equipment and corresponding coordination of logistics via planning and scheduling contributes to the success of earthmoving projects. On another perspective, poor scheduling may result in severe losses as pieces of equipment are idle and delayed due to constraints related to the working environment. Hence, equipment resources such as earthmoving machinery as well as topographical restrictions and parameters related to the scope of work must be considered during the planning and scheduling process. Presently, the scheduling of earthmoving activities is represented either by GANTT charts or by traditional networks, such as critical path and precedence diagram methods; meanwhile, linear scheduling provides an alternative to current scheduling techniques deployed for repetitive linear projects especially at their conceptual stage. Moreover, engineers and schedulers are in dire need of a decision support system that incorporates numerical analysis of earthmoving operations so that they can use it when making important decisions related to projects in hand. Based on the aforementioned, the main objective of this study is geared towards the deployment of an integrated expert system with a linear scheduling method for heavy earthmoving operations based on equipment fleet productivity rates at the conceptual design stage. Hence, an expert system comprising a knowledge base system and a hypothetical reasoning inference engine based on a forward chaining technique is presented. The expert system represents factual information pertaining to heavy earthmoving operations of infrastructure projects (i.e., static and dynamic parameters, besides working variables) where an inference engine is employed to evaluate 
the knowledge base and deploy subsequent numerical models and constraints to explicitly infer new results and input into the initial knowledge base. The deployment of the proposed system is expected to assist practitioners in the field of earthmoving operations in effectively identifying the time constraint related to this type of projects besides enhancing the capability of detecting potential scheduling conflicts at the conceptual design stage. Results presented in this study are anticipated to be very helpful to stakeholders and engineers.

\section{Literature Review}

Diverse methods and models have been proposed to schedule the operations of heavy earthmoving projects based on their diverse types of activities and associated equipment. However, these models are proposed for specific types of construction work due to many factors that contribute to the selection of equipment needed to do the work. Current models used for equipment selection utilize common methods such as (1) genetic algorithms; (2) simulation; (3) expert systems; (4) decision support systems; and (5) analytic hierarchy process, while some of the techniques used to determine equipment productivity are as follows: (1) bunching theory; (2) productivity curves; and (3) simulation. The majority of the studies published in the literature focus on optimizing the equipment selection needed in heavy civil work based on diverse parameters related to the scope of work; however, none of the studies were to include or perform operation analysis. One study conducted by Agrawal et al. [1] provided optimal algorithms to scheduling problems comprising linear workflows. Several subjective concepts such as latency and period minimization and bicriteria problems were addressed followed by the complexity results obtained. Song and Lee [2] presented a study of a stochastic lookahead scheduling method for linear construction projects. In their paper, traditional linear scheduling was taken one step ahead by integrating the scheduling of linear projects with performance data collected from earlier projects where a stochastic simulation model is designed and implemented by the authors. The main objective of their study was to forecast scheduling conflicts arising from uncertainties and provide corresponding productivity outputs. Moreover, König and Beißert [3] analyzed the optimization of construction scheduling by applying simulated annealing. In their study, a prototype that integrates simulated annealing with a constraint-based simulation model by utilizing a metaheuristic approach was presented. At the end, the authors used a case study to validate the developed prototype and to illustrate its capabilities in optimizing construction schedules. Linear scheduling has been utilized in numerous projects over the past decade; however, few studies did address the topic and did provide relative information on optimum scheduling techniques. Typically, scheduling forecasts at early planning stages are highly influenced by the subjectivity nature of the scheduler and the availability of information, whose results lead to a deterministic linear schedule. The main focus of the aforementioned studies has been geared towards the utilization of linear scheduling techniques during the operational phase rather than the early design stage such that scheduling uncertainties are not well addressed, which will result in major conflicts. In a study conducted by Srisuwanrat and Ioannou [4], an investigation of lead-time buffer under certainty using simulation and cost optimization was presented. Fluctuations in productivity rates within repetitive activities were analyzed and their corresponding impact on scheduling was illustrated. The authors mentioned that numerous studies had proposed diverse types of buffers that focused on halt-time rather than lead-time of a particular productivity line. In their paper, two diverse approaches, Sequence Step Algorithm (SQS-AL) and the Completed Unit Algorithm (CU-AL), were comprehensively studied via an implemented genetic algorithm (GA). Towards the end, leadtime buffer proved to reach optimum results resulting in reaping profitable gains. Although the proposed algorithm was of contribution to scheduling optimization techniques, productivity rates were assumed to be of static nature, which was an unrealistic representation of practical situations where fluctuations and uncertainties in production rates were inevitable. Likewise, Liu et al. [5] proposed a simulated GA system to determine linear scheduling optimum solutions. In their study, a simulated function of chaotic nature was proposed to predict uncertainties and interdependencies among temporal series of points in order to calculate corresponding productivity rates. At the end, results obtained from the GA were validated via the simulation system. Their study was of major significance and contributed towards simulated algorithms database for optimizing linear schedules; however, it is important to note that, typically, reliable data is collected from realistic cases as opposed to simulation systems to reach accurate productivity rates predictions at earlier design stages. Based on the aforementioned, few studies only considered numerous important factors for decision support systems; however, none of them had specifically viewed a project as a task or process that needs to be completed following definitive time constraints. Besides that, the majority of previous studies did not consider equipment operation analysis. Instead, the main focus was geared towards developing systems, algorithms, or frameworks in an attempt to assist the user in estimating project duration. Moreover, none of the studies had investigated numerical analysis of heavy earthmoving operations based on complex equipment performance parameters, which significantly impact the productivity rates of the selected equipment fleet at the conceptual stage. Therefore, in this paper, an integrated expert system that incorporates operational analysis with a linear approach is proposed to generate a linear schedule for heavy earthmoving operations at the conceptual design stage.

\section{Methodology and Development}

Operation analysis of selected types of equipment is essential for the development of the proposed integrated expert system. Seven major activities of earthwork will be considered: (1) clearing and grubbing; (2) excavating; (3) loading; (4) hauling; (5) backfilling; (6) grading; and (7) compacting, taking into consideration the variable factors that affect 


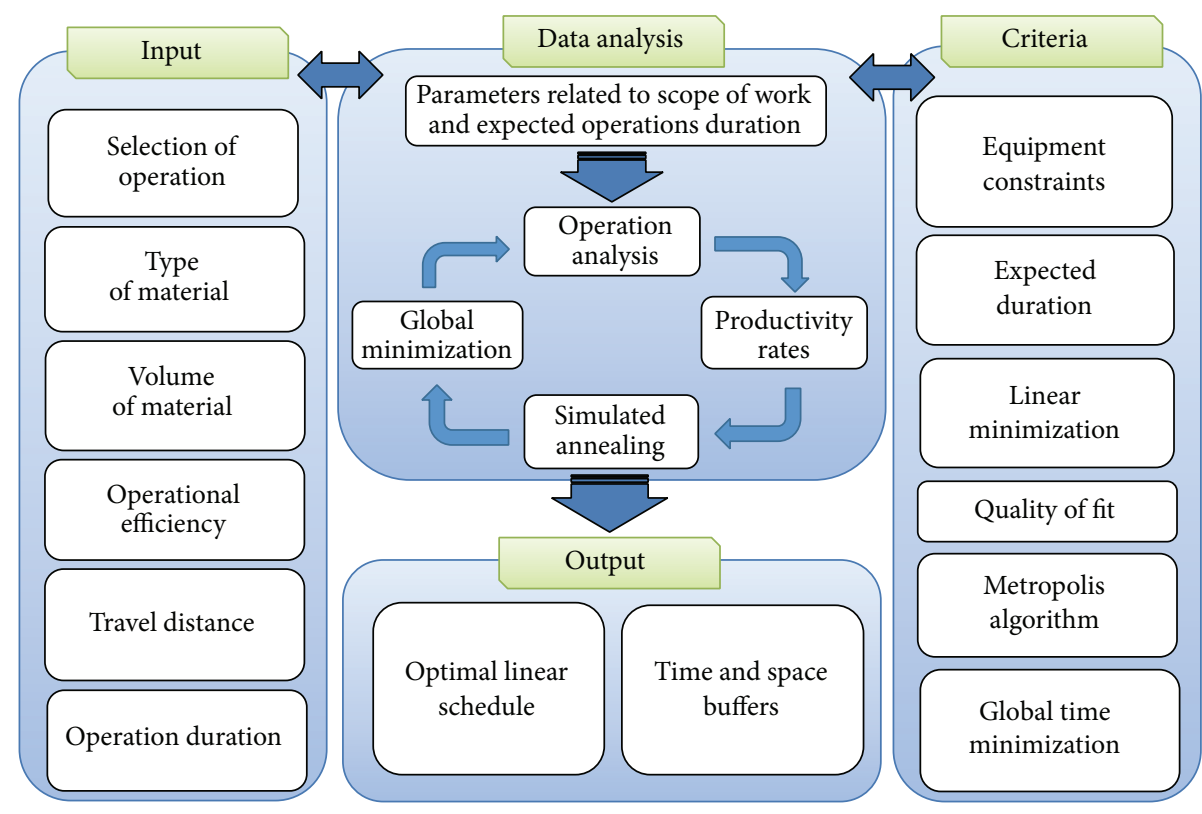

FIGURE 1: Proposed system architecture.

equipment productivity. The development of the system comprises the following five main steps: (a) data collection, such as site topography, soil characteristics, and equipment type; (b) numerical analysis of heavy earthmoving operations such as travel distance, rolling resistance, and cycle time; (c) determining productivity rates based on the user input parameters; (d) generating a linear schedule of earthmoving operations; and (e) minimal reduction of activities duration by applying local and global minimization algorithms. In order to conduct the analysis of earthmoving operations, a database of equipment type, capacity, and specifications besides site topographical and material characteristics is developed. Then, equipment productivity rates are calculated based on the user input parameters, including, but not limited to, soil characteristics, site topography, and volume of earthmoving while satisfying the following equipment constraints: rolling resistance, maximum rim pull, and horse power capacity. Following the determination of productivity rates, a linear approach to generating the schedule for heavy earthmoving operations based on a metaheuristic simulated annealing approach that utilizes a metropolis algorithm is deployed based on the calculated productivity rates for earthmoving operations as selected by the user. Figure 1 illustrates the architecture of proposed integrated expert system.

The proposed integrated system will be developed in an object-oriented .NET Framework and undertaken by the following five main tasks: (1) collect data related to the diverse processes of earthmoving operations and organize them into a database; (2) conduct numerical analysis for diverse equipment used for earthmoving operations and determine corresponding productivity rates based on a set of defined user input and the aforementioned parameters related to the scope of work; (3) analyze productivity rates data by fitting them into a distribution fitting and measure their corresponding quality of fit; (4) undertake the simulated annealing technique to determine the near optimum line of balance based on numerically expected activity durations; and (5) engage a global minimization approach to minimizing operations duration. Three main processes are categorized into the integrated expert system. The processes are as follows: (1) numerical analysis, which comprises an analytical linear algorithm that utilizes user input data and equipment specific parameters to numerically determine equipment fleet productivity rates. Afterwards, an analytical strategy is utilized for productivity rates to estimate operations durations based on the program evaluation and review technique (PERT); (2) linear scheduling process, which contains a constraint-based simulated approach for scheduling heavy earthmoving activities that is automatically linked to an output report that displays an instantaneous line of balance; and (3) time minimization process, which comprises a numerical technique that minimizes activity durations by seeking the global minimum value of the operations' expected durations.

3.1. Earthmoving Operations Analysis Phase. Selection and management of equipment fleet are a crucial task that considerably influences the success of an infrastructure project. This is to be much more critical in heavy earthmoving operations where equipment fleet plays a vital role in performing the work. In this type of projects, the equipment fleet represents the largest portion of the bidding price [6]. Consequently, practitioners in the field of infrastructure and construction industries understand the substantial impacts on their projects when equipment selection decisions are not made in a proper and timely manner. Therefore, the selection of fleet necessitates the commencement of a thorough economical numerical analysis of earthmoving operations at the conceptual stage. Since equipment selection is highly influenced by numerous factors, most practitioners tend to rely upon their 
experience and historical data in similar projects to assist them in determining the optimum fleet. Other approaches such as expert systems could be useful if only integrated with a database of historical data. These approaches are helpful for those who have been in the construction industry long enough and who are familiar with the variety and complexity of infrastructure projects. To overcome this shortcoming, the proposed system will be developed to integrate the collected data of selected equipment with an economic analysis method for diverse scopes of earthwork operations. The system will also be designed in a manner to provide users with a fleet that balances interdependent equipment such as the loadinghauling system. Although the proposed expert system will possess few limitations pertaining to equipment suppliers, resource availability, and earth rock material, it will be capable of assisting project stakeholders and practitioners in making decisions at the conceptual project stage. The objective of any project is to get the job done according to the specified timeframe and budget limitations. In order to achieve this goal, careful calculation of productivity rates for the fleet while considering the aforementioned diverse factors related to the scope of work is required. Schaufelberger [7] stated two general factors that must be considered in the process of selecting the equipment fleet: (a) cost effectiveness, which involves considering the equipment's size besides its proper type, and (b) versatility, which involves selecting equipment that can perform multiple tasks at the same time. In this paper, the aforementioned factors are incorporated into the operational analysis module. Afterwards, the operation analysis of equipment is undertaken to determine the constraints that must be satisfied. The first constraint is defined as the loaded weight. The loaded weight must not exceed the maximum allowable weight set by the manufacture. This constraint is expressed by using

$$
\mathrm{LW} \leq \mathrm{RW},
$$

where LW is the loaded weight and RW is the rated weight. The second constraint is defined as the total resistance. The total resistance must not exceed the allowable rim pull or drawbar if the equipment is wheel-mounted or crawlermounted, respectively. This constraint is expressed by using either (2) or (3):

$$
\begin{aligned}
& \mathrm{TR}<\mathrm{RP} \quad \text { if wheel-mounted } \\
& \mathrm{TR}<\mathrm{DB} \text { if crawler-mounted, }
\end{aligned}
$$

where TR is the total resistance, RP is the allowable rim pull, and DB is the allowable drawbar. If any of the abovementioned constraints is not satisfied, the system will automatically eliminate the equipment from the selection process. All of the aforementioned corresponding calculations are organized in different forms based on the equipment type to ease the development of the system. Following the determination of equipment constraints, productivity rates are calculated and extracted from the numerical analysis of earthmoving operations output database. The productivity rates are determined based on operational parameters categorized into four main groups summarized as follows: (1) spatial relationships, including (a) topography, (b) obstructions in earth excavation, (c) clearance heights, (d) required heights, (e) required reach maneuverability, and (f) location of hauling units; (2) soil characteristics, including (a) rolling and grade resistances, which provide traction and soil stability, (b) potential changes in characteristics during performance of work, (c) required force to loosen materials, (d) need for ripping and pushing attachments, and (e) abrasiveness and other rough earth qualities, such as rock material that may cause problems to equipment; (3) contract provisions, including (a) quantity of earth involved, (b) time constraints and weather conditions, (c) requirements for payment and subsequent cash flow, (d) legal constraints on the weight and size of equipment, and (e) other restrictions, such as traffic, hours, dust, and noise; and (4) logistical considerations. including (a) availability of required equipment and their operators, (b) mobilizing and demobilizing time and cost, (c) use of equipment in preceding operations and idle time, (d) economical equipment costs, and (e) support facilities. In this paper, the estimation of productivity rates is performed for each type of equipment individually. Productivity rates, which are determined based on equipment specifications data and specific parameters related to the location (i.e., soil type, traveled distance, altitude, and job conditions), are calculated by using the following equation:

$$
\text { Productivity }=\frac{\text { Volume of earth } * \mathrm{OE}}{\text { Cycle time }},
$$

where volume of earth is the required amount of earthmoving operation (bank cubic yards), OE is the operational efficiency ( $\mathrm{min} / \mathrm{hr}$ ), and cycle time is the time needed to complete an earthmoving operation (minutes), which is the total of fixed and variable times and includes the time needed to complete one cycle while being empty and/or loaded depending on the type of equipment. In this study, empty and/or loaded times of equipment are determined based on the manufacturer, Caterpillar ${ }^{\circledR}$, performance charts.

3.1.1. Estimating Activity Duration. Estimating the duration of earthmoving activity is a multifaceted task that faces engineers and schedulers as the occurrence of unforeseen events is very probable and unpredictable. In this paper, a linear scheduling technique that encompasses the capability of modeling the variability of earthmoving operations performance-related factors which significantly affect productivity data is proposed as follows: (i) weather, which is taken into account within the topography, variability in soil saturation and density, and altitude parameters; (ii) learning curve, which is taken into account within the equipment cycle time, tire penetration, and operational efficiency parameters; (iii) overtime, which is taken into account within the rolling and grade resistances as they influence the productivity rate and accordingly the operator wage parameters; (iv) space congestion, which is taken into account within the clearance heights of adjacent structures, reach maneuverability, and obstructions in earth excavation parameters; and (v) sub/superstructure design changes, which are taken into account within the availability of equipment and operators for high 
risk operations, equipment capacity, and volume of earthmoving parameters. In order to provide a simulation input for a scheduler, a fleet selection system developed earlier by the authors of this study for the selection of fleet based on their productivity rates is utilized to implement into the proposed linear scheduling system [8-10]. Earthmoving operations and their corresponding productivity rates and most likely durations are automatically extracted from the database of the fleet selection system in order to generate a histogram where a statistical normal distribution is utilized to quality-fit the productivity rates by utilizing the Gaussian equation (5) as follows:

$$
f(x, \mu, \sigma)=\frac{e^{-(x-\mu)^{2} / 2 \sigma^{2}}}{\sigma \sqrt{ } 2 \pi}
$$

where $x$ is the random variable, $\mu$ is the mean, and $\sigma$ is the standard deviation. The process begins by normalizing the normal distribution of productivity rates. Afterwards, distribution parameters are determined throughout the fitting process. Then, the expected duration of each earthmoving operation is calculated by using

$$
E=\frac{(O+4 M+P)}{6},
$$

where $E$ is the expected duration time, $O$ is the optimistic duration time, where it is estimated to be the shortest duration, $M$ is the most likely duration time, and $P$ is the pessimistic duration time.

3.1.2. Normal Distribution Fitting. The initial step when modeling normal distribution fittings is to develop a generic histogram pertaining to earthmoving operations productivity rates. Based on the characteristic shape of the generated histogram, an indication of a standard distribution that will result in a "best-fit" fitting will be evident. Furthermore, corresponding distribution parameters, such as the mean and standard deviation, may be determined from the distribution fitting. It is important to note that, due to the technological advancement in normal distribution modeling software, an indefinite number of distributions may be automatically fitted within a reasonable time frame. However, a selective number of candidate distributions are typically forwarded to quality of fit testing procedure prior to engagement in further processes.

3.1.3. Quality of Fit. Although multiple standard distributions are capable of modeling a distribution fitting, significant variance may be noticed in the manner that they represent the actual distribution underlying histogram data set. Testing the quality of fit for a distribution is conducted by either of the two following procedures: (1) heuristic, where manual inspection is conducted in parallel with an error minimization procedure, or (2) nonheuristic procedure, where hypothetical procedures such as the chi-square test are deployed [2]. In order to ease the use of distribution modeling among owners and designers, the manual inspection of distribution fitting with an error minimization procedure is adopted since distribution fittings are automatically generated with advanced modeling software available in the market. In this study, productivity rate histograms are normalized with the suggested scaled distribution. As part of enhancing the quality of fit, an error minimization procedure is proposed for the curve-fitting of the suggested distribution to the actually obtained one. The procedure is based on applying a scaling factor to the suggested one. Sum $(E)$ of the squares of differences between the actual and suggested fit is then minimized to obtain the magnitude of adjustment factor that results into the best fit with actual data. The error minimization procedure is identified as

$$
E_{\min }=\sum_{i=1}^{n}\left[\frac{\left(p_{\mathrm{act}, i}-a\left(p_{\mathrm{sug}, i}\right)\right)}{\bar{p}_{\mathrm{act}, i}}\right]^{2},
$$

where $E_{\min }$ is the minimized error, $i=1, \ldots, n$ is the number of actual productivity rates, $p_{\text {act, } i}$ is the actual productivity rate at $i$ th location, $p_{\text {sug }, i}$ is the suggested productivity rate at $i$ th location, and $a$ is a scaling factor to be applied to the suggested productivity rate values. It is noted that the bracketed terms in (7) have been normalized with respect to average actual productivity rate, $\bar{p}_{\text {act }, i}$, as

$$
\bar{p}_{\mathrm{act}, i}=\frac{1}{n} \sum_{i=1}^{n} p_{\mathrm{act}, i} \text {. }
$$

Towards the end, it is important to note that the suggested productivity rates distribution fit provides a good representation of a logically sequenced line of balance where time and space buffers are respected.

3.2. Linear Scheduling Phase. A particular earthmoving activity is presented in linear scheduling as a linear production curve such that quantities in loose cubic yard (LCY) and duration in days are on $y$ - and $x$-axes, respectively, and are interrelated in 2D graphical display. Earlier studies have shown that linear scheduling method may be comprehended as a specialized construction scheduling alternative tool for phased repetitive operations that provides a visualization of scheduling information rather than the traditional scheduling methods, such as GANTT charts, in terms of relating earthmoving operations and production rates to time and space related constraints. However, it is worth noting that, typically, all operations are considered to be critical in linear scheduling and work continuity must be maintained consistently throughout the project in order to achieve efficient heavy earthmoving operations. Authors were not able to find studies that address the major pitfall of determining effective linear scheduling solutions for heavy earthmoving operations. In this paper, efforts will be focused on designing, implementing, and enhancing earlier relative work pertaining to optimizing linear schedules in heavy earthmoving operations as follows: (1) unifying the process of maximizing fleet productivity rates while taking into account temporal variability; (2) satisfying time and space related constraints; and (3) modeling an integrated simulated approach for determining near optimum linear schedules. 


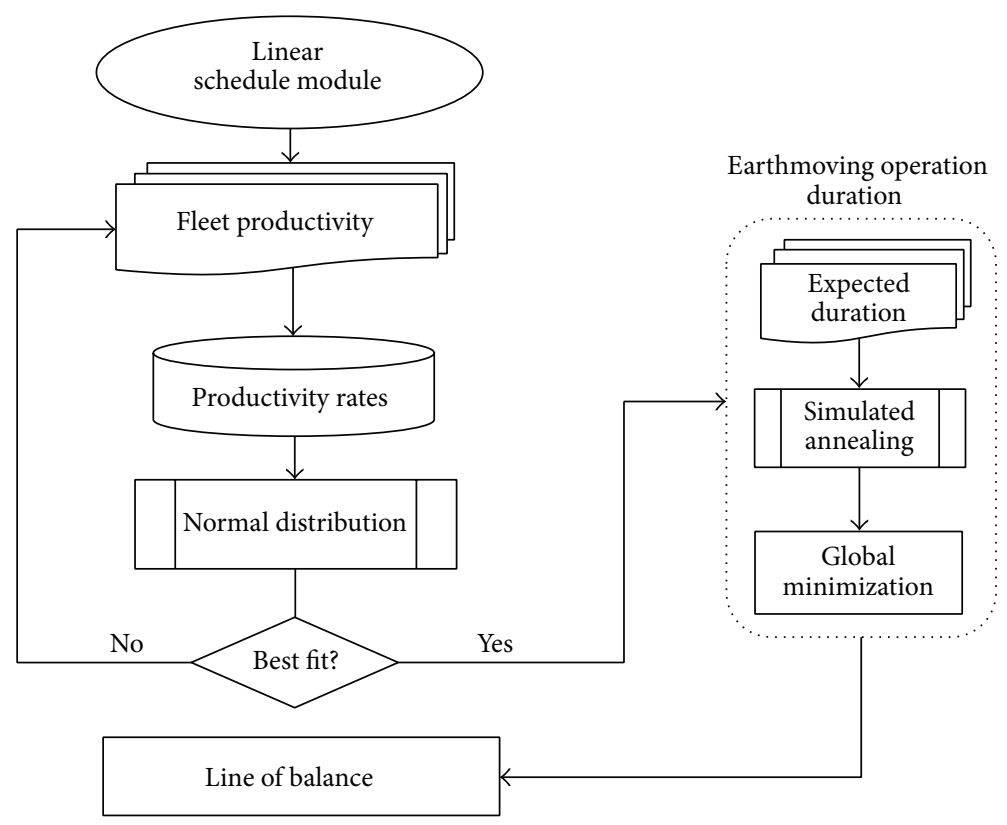

Figure 2: Linear schedule system overview.

In this regard, linear scheduling is deployed as a scheduling forecasting technique that permits a scheduler to balance the productivity rates and to maintain work continuity while refraining from idle production resources at the conceptual design stage of an earthmoving project life. Figure 2 provides an overview of the process to develop the proposed linear schedule system for earthmoving operations.

The overall process underlies the determination of equipment productivity rates by undertaking a numerical analysis of equipment operations based on a linear approach. Once determined, productivity rates are fitted into a Gaussian distribution fitting to measure its corresponding goodness of fitting. If accepted, the process automatically proceeds to the successive process to estimate operations durations by deploying (5) and (6) followed by the metaheuristic simulated annealing algorithm to generate a linear schedule at the conceptual stage. At last, a global duration minimization approach is deployed to minimize the duration of the earthmoving operations.

\section{Cost Minimization Phase}

The main objective of the minimization function used in this study is to maximize productivity by minimizing equipment fleet costs, where linear programming is the methodology used for determining the equipment selection. In this study, a fleet is defined as a set of selected equipment that will yield to the least ownership and operating costs. However, since owning and operating costs are inversely proportional to equipment operation analysis, the fleet is selected based on its productivity rate and cost. For example, if a particular equipment fleet has the maximum productivity rate, it is going to yield the least owning and operating costs and vice versa. The fleet is obtained by using the cost minimization approach in a given mathematical model for a list of requirements and constraints represented as linear relationships. All constraints obtained from the operation analysis are represented in a mathematical form and incorporated into the cost minimization system. These constraints, however, limit the degree to which the objective function can be pursued. The following expression is then used to select the equipment with the minimum unit cost: $\mathrm{MIN}=$ $\sum_{i=1}^{n} \mathrm{EU}$, where $i$ is the amount of equipment and EU is the equipment unit cost. It is important to note that the proposed system accounts for a time-cost tradeoff that occurs during the selection process. In other words, the proposed system is designed in a manner to extract the most likely activity duration inputted by the user at the fleet selection system and compares it with the required time based on the volume of earth material involved and corresponding productivity rates. For example, if the operation duration data inputted by the user is less than the required time obtained from the proposed system calculations, the system will select a fleet with much higher costs and vice versa. Figures 3 and 4 summarize the development process of the cost minimization function and fleet selection, respectively.

4.1. Uniform Dependence Algorithms. In order to determine a near optimum linear schedule, a duration domain to execute a task must be defined. A unique class of algorithm termed as uniform dependence algorithm is of significance in parallel minimization problems. In this study, uniform dependence algorithms are deployed for determining a near optimum linear schedule. A uniform dependence algorithm may be represented in accordance with Shang and Fortes [11] expressions:

$$
\begin{aligned}
u(t) & =y_{t}\left(u_{t}\left(t-d_{1}\right), \ldots, u_{t}\left(t-d_{i}\right)\right) \\
\text { while } t \in T ; T=\{x: A x \leq b\} ; T \equiv(A, b), & \\
D & =\left(d_{1}, d_{2}, \ldots, d_{m}\right) \quad \text { while } i=1, \ldots, m ; m \geq 0,
\end{aligned}
$$




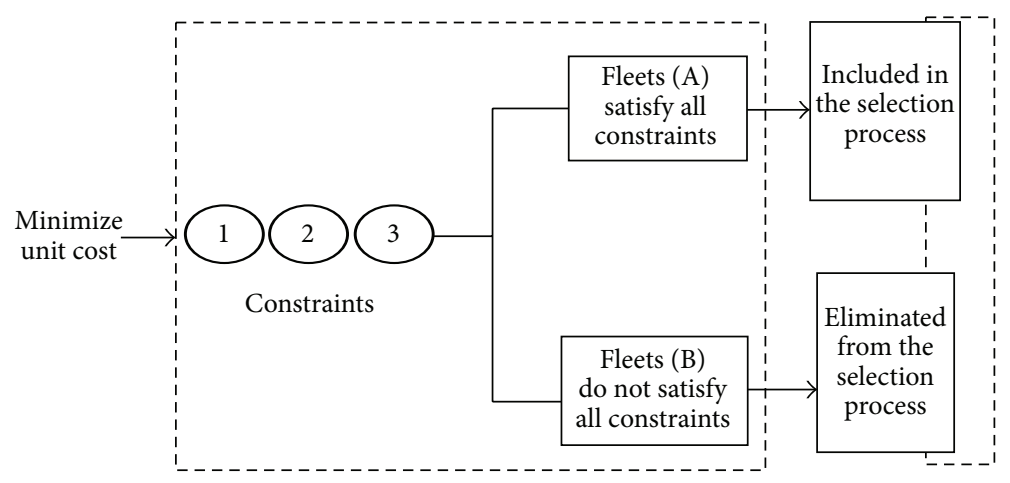

Figure 3: Cost minimization process flow.

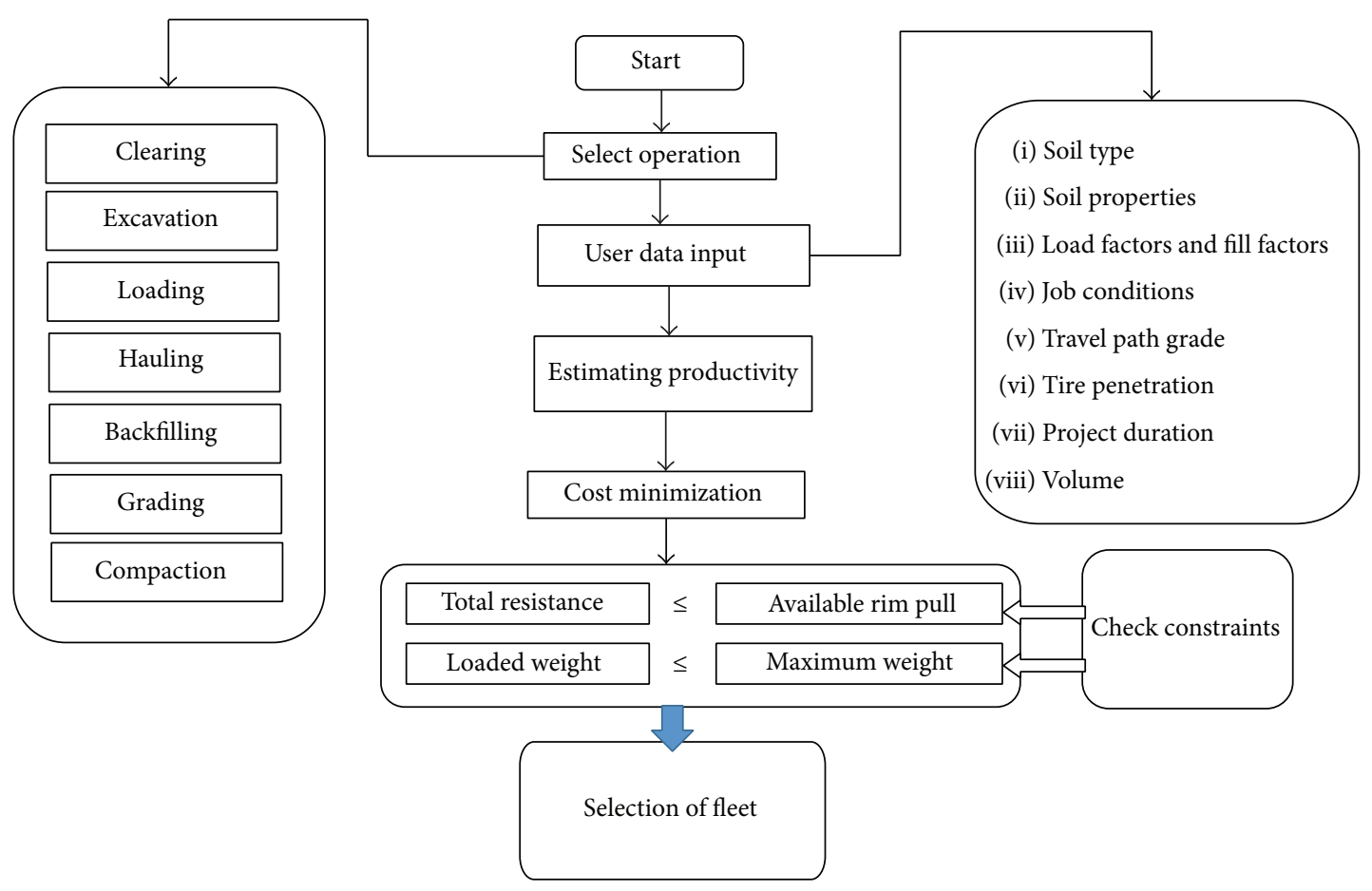

FIGURE 4: Linear programming process flowchart.

where $i$ is an integer, $t$ is an index point, $T$ is the index set of vectors, $y_{t}$ is the numerical computation at point $t, u_{t}$ is the time-value function at point $t, A$ is matrix of dimension $(a * n), a$ is the number of constraints, $n$ is the dimension of the domain, $b$ is the domain constraints, $D$ is dependence matrix $(n * m), d_{i}$ is the dependence matrix vector, and $m$ is the number of dependence vectors. In this study, a uniform dependence algorithm of $\mathrm{ALG}=(T, D)$ is applied to determine a linear schedule such that $A$ is constraint matrix $(a * n)$, where $a$ is the number of time and space buffer constraints, $n$ is the dimension of the domain, and $D$ is dependence matrix $(n * m)$, where $m$ is the number of dependence vectors. The two index points, $t_{1}$ and $t_{2}$, are assigned to two dependent activities such that $t_{1}<t_{2}$ and $t_{2}=t_{1}+d_{i}$ for $d_{i} \in D$, where $d_{i}$ is the dependence matrix vector and points $t_{1}$ and $t_{2}$ are of the index set, $T$, such that $t_{2}$ is dependent upon $t_{1}$. A linear schedule for the uniform dependence algorithm is then established as a mapping function, $\sigma_{\Pi}$, such that, for a random index point, where $\sigma_{\Pi}(t)=\left[\Pi_{t}+c\right], \Pi$ is the linear schedule vector, which is subject to constraints $t \in T, \sigma_{\Pi}\left(t_{1}\right)<\sigma_{\Pi}\left(t_{2}\right)$, and $\Pi D \geq 1$ and time buffer constraint $c=-\min (\Pi t, t \in T)$, which is offset (c) [11]. Upon satisfying the aforementioned conditions, a linear schedule is then acceptable along with its corresponding dependencies. In other words, the process of determining a linear schedule is commenced by assigning time and space buffer constraints to develop constraint matrix $(a * n)$. Following the determination of the constraint matrix, dependence matrix $(n * m)$ is developed to represent the number of dependent scheduling alternatives. 
4.2. Linear Schedule Minimization Process. In order to properly minimize the durations of earthmoving activities, one must understand all related constraints; otherwise, failure to do so may lead to erroneous results. In scheduling terms, deployment of equipment resources is a multifaceted task for engineers and construction planners. In this study, an earthmoving linear scheduling system is proposed to minimize the use of available equipment by maximizing fleet productivity rates through the selection of the most suitable equipment for an earthmoving operation. The system utilizes simulated annealing as a minimization tool by utilizing expressions (9) and supported by a classical constrained-based simulated algorithm defined by the space buffer constraints $t \in T$, $\sigma_{\Pi}\left(t_{1}\right)<\sigma_{\Pi}\left(t_{2}\right)$, and $\Pi D \geq 1$ and the time buffer constraint $c=-\min (\Pi t, t \in T)$.

4.3. Simulated Annealing. Simulated annealing is a strategic approach that may be utilized to solve scheduling conflicts. In metallurgic industries, annealing processes are the main heat treatment operations. In this study, simulated annealing is utilized in a similar manner in order to determine a linear schedule for earthmoving operations. Typically, when a metallic substance undergoes annealing, it is heated to the maximum temperature where it reaches the limit of liquefaction. Afterwards, it cools down gradually to form the desired solid shape and corresponding chemical characteristics. The final status of the substance is highly dependent upon the cooling methodology implemented. For instance, if the cooling procedure is done quickly, the substance is brittle and heterogeneous. On the other hand, if the cooling process is controlled gradually, the substance status is ductile and homogenous. Typically, simulated annealing algorithm is undertaken in three main phases as follows: (i) perturbation of solution; (ii) evaluation of quality; and (iii) acceptance of solution. An initially high temperature is set and utilized to plan the perturbation, evaluation, and acceptance of final solution by gradually decreasing the temperature via a defined numerical function. With simulated annealing, an enormous amount of random numbers is generated, depending on user discretion on whether quality or speed of solution or both are desired. In this study, the quality of linear schedule is crucial and therefore the algorithm is implemented accordingly [12].

4.3.1. Constraint-Based Simulation. Beißert et al. [13] summarized few constraints for typical earthmoving projects as follows: (1) technological advancements; (2) equipment capacity; (3) availability of resources; and (4) logistical aspects. It is important to note that constraints parameters, specified among a defined set of variables, must be satisfied for one activity prior to proceeding to the next one. In addition to that, for heavy earthmoving operations, the two major constraints that must be satisfied are (1) time- and (2) space-related constraints, which are typically referred to as time and space buffers, respectively. In this study, efforts to enhance the discrete event simulation technique will be utilized such that constraint satisfaction problems are integrated with a simulated annealing approach such that interdependencies among tasks, that is, loading-hauling, are taken into consideration and results are obtained in a timely manner. In simulated annealing processes, the presumed constraint is always satisfied and controlled within the procedure itself. In other words, no event or task can be scheduled without its time and space buffers being met, which produces a logically sequenced linear schedule where further analysis to time and space constraints may be conducted.

4.3.2. Metropolis Algorithm. In this context, a metaheuristic metropolis algorithm is deployed as a local minimization approach to resolve linear scheduling combinational conflicts and reach a near optimum solution within reasonable cycle time. The process begins when earthmoving operations unveil their original state and reconfigure into a highly organized structure with lower energy than the initial state. In this study, the simulated annealing procedure may be comprehended as the methodology deployed to reach at a near optimum solution, which represents the possibility of new configurations of the earthmoving operations. Simulated annealing is usually implemented by assuming a high initial duration and determines via a metaheuristic approach a new solution within the neighborhood of the initial solution. It is important to note that the probability of acceptance of reconfigured solutions is dependent upon the difference between immediate consecutive solutions $(\varepsilon)$ and duration $(\alpha)$. Once accepted, the new solution is set as the starting point for the consecutive minimization cycle. Consequently, in order to implement the generic metropolis algorithm, the following criteria must be met: (a) suitable neighborhood; (b) proper probability of acceptance; and (c) effective duration decreasing rate in order to reach a successful near optimum linear schedule and consequently escape local minima [14]. Towards the end, simulated annealing is integrated into the constraint-based simulation in an attempt to enhance neighboring schedules by substituting operations. Once a near optimum linear schedule is determined, the newer solution replaces the older one and simultaneously enables formerly declined solutions to be accepted in order to escape local minima.

4.3.3. Neighborhood Sequencing. In simulated annealing scheduling approaches, the distinctive concept of local "neighbor-hooding" is crucial. Conceptually, a schedule possesses a local neighborhood only when two operations of the same ranking are substituted. Typically, tasks ranking is determined through a topological sorting technique. The concept of topological sorting is based on transforming a partial order to a total order. For instance, if $a>b$ and $b>c$, then it implies that $a>c$. Hence, the system proceeds by generating multiple solutions by substituting operations of local neighborhood with similar rankings. Once a solution is generated, neighborhood operations are assigned ranks along with a corresponding execution order for each ranked operation. For instance, operations $A$ and $B$ may be assigned rank one; however, operation $B$ may precede operation $A$ in terms of order of execution. In the next step, operations $C$ 
and $D$ of rank two may be substituted to form an alternative solution and so forth.

4.3.4. Rule of Acceptance. Rules of accepting a solution within a simulated annealing process is an important element of the generalized metropolis algorithm. In order to illustrate probability of acceptance process, a system in its current state described by $N$-dimensional vector $(x)$ comprising a probability function $f(t)$ is defined. Then, a set of values that control the convergence speed of an annealing algorithm must be defined and can never be predicted at the initial stage. Instead, the values depend on the type of minimization problem and must be adjusted accordingly. Therefore, the process commences upon the selection of initial configuration, $x$. Then, the initial number of simulations $\left(n_{s}\right)$ must be set to null. Afterwards, initial duration $\left(t_{0}\right)$ must be set to some high value. This step is considered to be one of the most significant steps as the convergence of the simulated annealing algorithm is dependent on the selection of initial duration, $t_{0}$, convergence parameter, $\alpha$, and decreasing rate, $\Delta t$. Within the scope of this study, the decreasing rate of the initial duration is determined as per Tobochnik and Gould $[12]$ in

$$
\Delta t=t_{i}(1-\alpha),
$$

where $\Delta t$ is the decreasing rate, $t_{i}$ is the current duration, and $\alpha$ is the convergence parameter. According to Tobochnik and Gould [12] and based on earlier studies typical values of the convergence parameter, $\alpha$, vary between 0.8 and 0.999 with the latter being the closest to ideal. Prior to decreasing in duration, an initial value of the probability function must be obtained as per Tobochnik and Gould [12] in

$$
f_{a}=f\left(t_{i}\right) \text {. }
$$

Then, a second value is determined following the transition as per Tobochnik and Gould [12] in

$$
f_{b}=f\left(t_{i}-\Delta t\right) .
$$

Afterwards, the difference between the obtained values of the probability function is determined according to Tobochnik and Gould [12] in the following equation:

$$
\Delta f=f_{b}-f_{a} .
$$

The following step involves an "if-then" loop console where if $\Delta f \leq 0$, the current state is accepted; otherwise, generic value, $\varepsilon$, is the value at which the algorithm is terminated. In this study, $\varepsilon$ is assigned a value of 0.001 . The rule of accepting the state of function is then defined as per Tobochnik and Gould [12] in

$$
\varepsilon<e^{-\Delta f / t_{i}},
$$

where $\varepsilon$ is the value at which the algorithm is terminated, $\Delta f$ is the change in probability functions values, and $t_{i}$ is the current duration. However, if the state of the function is rejected, then the process is directed to another transitional iteration, $n_{s}=n_{s}+1$, until the initial duration reaches the value of zero. The detailed procedure for rules of accepting a function state as adopted from Tobochnik and Gould [12] is summarized in Figure 5.

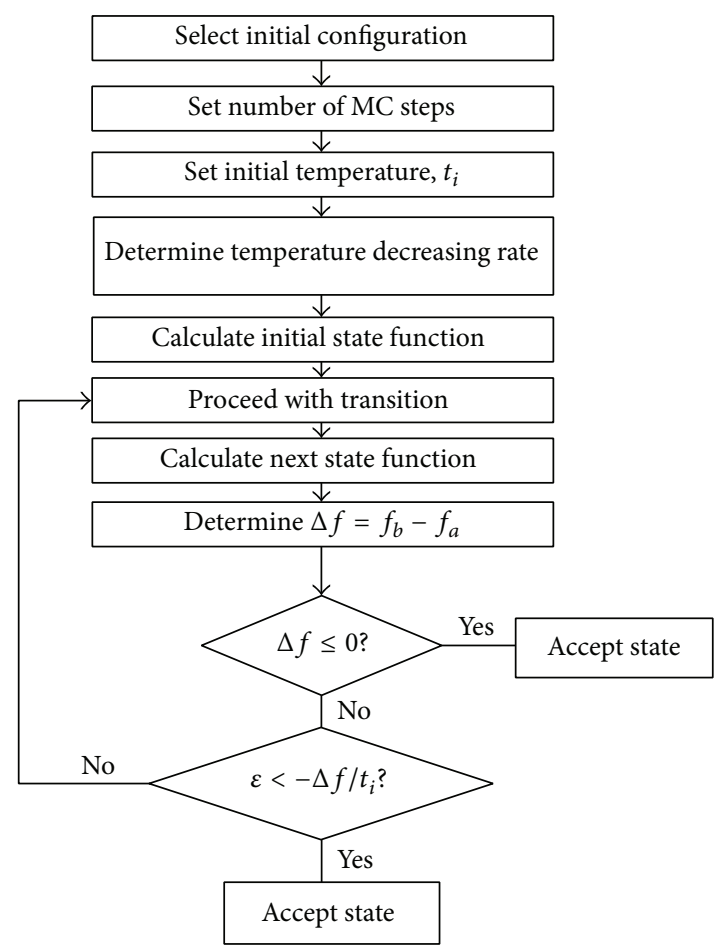

FIGURE 5: Simulated annealing process.

4.4. Global Duration Minimization. Global duration minimization is an analytical procedure where the solution of an objective polynomial function is determined by obtaining the global minimum value. Global duration minimization is known for its capability of bypassing local minima and seeking a global solution of a boundary-constrained polynomial function. In this study, efforts are focused to enhance the linear scheduling technique a step further by proposing a global minimization procedure in order to minimize the duration of earthmoving activities while respecting time and space buffer constraints. Therefore, a uniform dependence algorithm of ALG $=(T, D)$ is defined such that $\sigma_{\Pi}$ is the linear schedule. The overall project duration is calculated as per Shang and Fortes [11] in

$$
\begin{aligned}
& T_{\Pi}=1+\max \left(\sigma_{\Pi}(t), t \in T\right) \\
& T_{\Pi}=1+\max \left(\left[\Pi_{t 2}\right]-\left[\Pi_{t 1}\right], \text { where } t_{1}, t_{2} \in T\right) .
\end{aligned}
$$

\section{System Implementation}

The implementation of simulated annealing algorithm is undertaken in two main steps: (i) perturbation and (ii) quality evaluation. The algorithm is implemented in such a way that the error determined from the preceding solution is utilized to determine the acceptance of the new solution. At first, near optimum problem solutions of $X$ variables are defined as per Ledesma et al. [15] in

$$
X=\left\{x_{1}, x_{2}, x_{3}, \ldots, x_{M}\right\}
$$


where $X$ is the near optimum solution and $x_{1}, x_{2}, x_{3}, \ldots, x_{M}$ are simulated annealing solutions to a linear scheduling problem. Afterwards, the algorithm duration-gradient process is established as per Ledesma et al. [15] in

$$
T=T_{1}, T_{2}, T_{3}, \ldots, T_{N}
$$

where $T_{1}$ is the initial duration; $T_{N}$ is the final duration; and $N$ is the number of durations. The selection of initial duration for the simulated annealing approach is crucial as it significantly influences the scheduling of earthmoving operations. Duration values are discrete values selected based on a definitive convergence-gradient algorithm, which will be presented later. In order to enhance simulated annealing efficiency, a definitive number of iterative processes at each duration level are generated as per Ledesma et al. [15] in

$$
X=\left\{x_{1, i}, x_{2, i}, x_{3, i}, \ldots, x_{M, i}\right\}, \quad \text { where } i=1,2,3, \ldots \text {, }
$$

where $i$ is the number of perturbations and $x_{1, i}$ is the value of $x_{1}$ following $i$ th perturbation. Hence, the number of perturbations at the end of each duration level is of value $K$ such that $X_{k}$ is the solution to the problem at the end of $T_{i}$. Generally, an error is always associated with each of discrete solutions, $X_{i}$. Therefore, $E_{1}, E_{2}$, and $E_{3}$ are errors of corresponding solutions $X_{1}, X_{2}$, and $X_{3}$, respectively. Following the determination of solution errors, the evaluation of solution quality and acceptance of the perturbation process is deployed. The metropolis algorithm is then implemented in order to determine the probability of accepting a perturbed solution as per Ledesma et al. [15] in

$$
R_{a}= \begin{cases}e^{-k \Delta E / T} & \Delta E>0 \\ 1 & \Delta E \leq 0,\end{cases}
$$

where $R_{a}$ is the rule of acceptance; $\Delta E$ is the solution error obtained due to the difference in solution before and after perturbation; $T$ is the set duration; and $k$ is a constant determined based on initial duration and its associated error as described in the following section. It was found that at very high durations, the algorithm is susceptible to accepting any solution. However, as the duration gradually decreases, the annealing process becomes more effective and adapts a selection methodology of the perturbed solution highly dependent upon the error value. Based on the aforementioned, the duration-gradient process is controlled by gradually decreasing the duration resulting in a timeconsuming annealing process. On the other hand, perturbed solutions along with high probability of acceptance and enhanced quality are obtained. In this study, the constant, $k$, is carefully chosen in an attempt to save the time consumed for the annealing process. For instance, if $k$ equals $T$, then the algorithm is susceptible to accepting solutions with higher probability of acceptance along with higher corresponding errors values as well. On the other hand, as the ratio of $k$ to $T$ approaches unity at the initial stage, an enhanced perturbed solution with a higher probability of acceptance and lower corresponding error value is accepted.
5.1. Estimation of $k$-Value. The estimation of $k$-value is crucial at the initial stage of the simulated annealing process. As stated earlier, the efficiency of the simulated algorithm is highly dependent upon the set of acceptance criteria such as difference in errors and rule of acceptance. A good estimation of $k$-value results in substantial minimization of computational time. Prior to commencement of the annealing process, initial value $X_{0}$ is created based on (18) along with its corresponding error, $E_{0}$. Afterwards, $k$-value is determined by following an iterative process as per Ledesma et al. [15] in equations (20) and (21) as follows:

$$
\begin{aligned}
\Delta E & \approx \frac{1}{Q-1} \sum_{i=1}^{Q} E_{i}-\frac{1}{Q(Q-1)} \sum_{i=1}^{Q}\left(E_{i}\right)^{2}, \\
k & =\frac{T_{0} \ln (0.8)}{\Delta E},
\end{aligned}
$$

where $\Delta E$ is the difference in errors estimate; $Q$ is the number of perturbations; and $T_{0}$ is the initial duration. It is important to note that the value of $\Delta E$ is an estimate value at the initial stage as shown in (20). Upon determining the value of $\Delta E, k$-value is determined in accordance with (21). Once determined, $k$-value is substituted into (19) to determine acceptance value in order to accept activities' durations and determine a near optimum line of balance.

5.2. Fleet Selection System. In this study, the proposed fleet selection system comprises earthmoving modules with the following seven major operations: (1) clearing and grubbing; (2) excavating; (3) loading; (4) hauling; (5) backfilling; (6) grading; and (7) compacting operations. The proposed fleet selection system gateway is illustrated in Figure 6.

As shown in Figure 6, once the user selects the desired earthmoving operation, the proposed system displays an input form where users input necessary parameters related to the scope of work. Within each operation, input forms that include common parameters to diverse equipment capable of undertaking the selected earthmoving operation are automatically extracted and imported into the database in order to proceed with the numerical analysis of the earthmoving operation as illustrated in Figure 7.

Afterwards, the user must enter project specific data required to execute necessary calculations. It is important to note that the data required for the numerical analysis of earthmoving operations vary from one operation to the other; however, (1) material density, (2) fill factors, (3) safe factors, (4) time constraints, (5) volume, and (6) operational efficiency are a common list of parameters among the earthmoving operations. The volume of earth material to be excavated/hauled is measured in terms of bank cubic yard and is inputted by the user, which is in turn utilized for the numerical analysis of the earthmoving operation to obtain the necessary fleet and corresponding productivity rates. In case equipment exceeds system constraints, a popup message notifies the user of the equipment models that will not be considered in the fleet selection process. Then, the user is directed to the operation analysis process in order to obtain fleet productivity rates. Once equipment productivity 


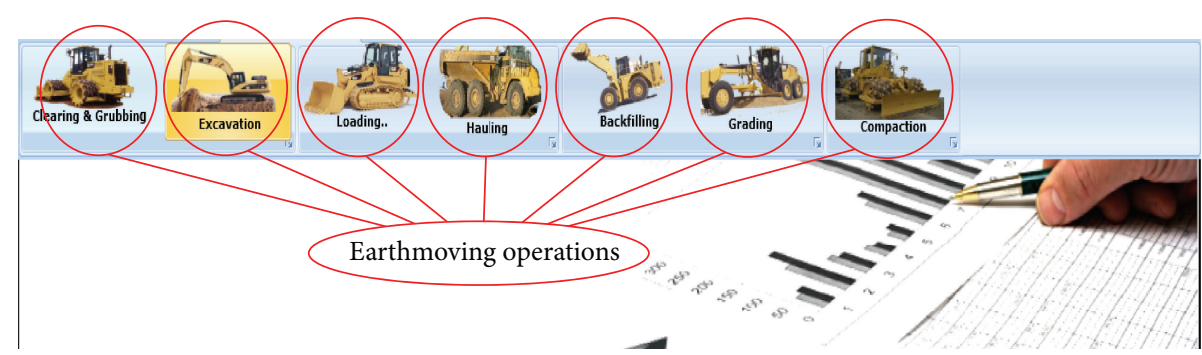

FIGURE 6: Fleet selection system gateway.

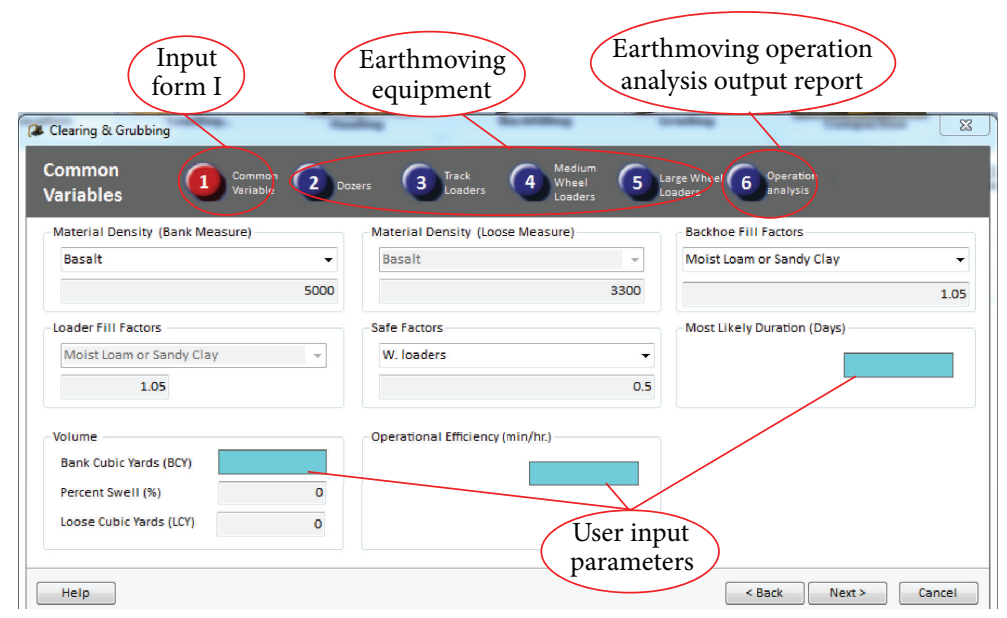

FIGURE 7: Clearing and grubbing operation input form I.

rates are determined, the user will select the equipment model desired along with its corresponding productivity rate. Also, the user may select the amount of equipment required to complete the earthmoving operation.

5.3. Earthmoving Linear Schedule System. Once completed, the system then automatically extracts the selected productivity rates from the fleet selection system and imports them into the earthmoving linear schedule system. The productivity rates extracted from the fleet selection system are obtained in LCY/hr (loose cubic yards/hour). The daily output in LCY (loose cubic yards) is then estimated based on the assumption of eight working hours per day. Moreover, the most likely duration in days to complete an earthmoving operation is automatically extracted from the fleet selection system and imported into the earthmoving linear schedule system. The user is then invited to input optimistic and pessimistic durations in days to complete the earthmoving operation. It is important to note that the proposed system possesses one limitation pertaining to optimistic and pessimistic durations of earthmoving operations, which are mainly influenced by the scale of subjectivity involved, and is highly dependent upon user experience on similar projects. In order to overcome such limitation, the proposed system incorporates the program evaluation and review technique (PERT) estimation equation (6), which accounts for the time variability in the duration of an earthmoving operation and determines its expected duration accordingly. On the other hand, the system may be utilized as an inference engine for backward reasoning in order to determine optimistic and pessimistic durations of an earthmoving operation when the linear schedule is "predetermined" at the conceptual stage. Once expected durations are obtained, productivity rates are automatically organized into a histogram where a quality of fit is processed as presented in Section 3.1.3. Once completed, the user may proceed by setting an initial duration and rule of acceptance parameters as discussed in Section 4.3.4.

\section{System Validation}

To validate the workability of the proposed system, an actual project that consists of heavy earthmoving operations for an earth fill project along with their corresponding durations is recalled from the literature [16]. The challenge underlying the system validation is to determine the near optimum fleet necessary to execute the earthmoving operations. The project was phased into three stages, each at different elevation and spanning a complete construction season. In this study, however, the scope of earthwork at operational stage I is used to validate the proposed system. Table 1 summarizes the project fill earthmoving parameters for earthmoving operations stage I.

Prior to inputting data, a list of assumptions is made based on normal job conditions as follows: (1) gear efficiency is 0.85 ; 
TABLE 1: Project fill parameters for earthmoving operations stage I*.

\begin{tabular}{lccc}
\hline \multicolumn{2}{l}{ Soil type Volume (BCY) } & Haul distance (ft.) & Amount of equipment \\
\hline Moraine & 29,200 & 49,842 & $(6,1,1,1)^{* *}$ \\
Granular & 14,500 & 77,732 & $(6,1,1,1)^{* *}$ \\
Rock & 192,700 & 10,150 & $(8,1,1,1)^{* *}$ \\
\hline
\end{tabular}

Source: [16].

${ }^{*}$ Clearing and grubbing, excavation, loading, hauling, backfilling, grading, and compaction.

${ }^{* *}$ Hauling, loading, grading, and compaction.

(2) operational efficiency is $50(\mathrm{~min} / \mathrm{hr})$; (3) job conditions are average; (4) altitude is 2,500 ft.; and (5) tire penetration is $3 \mathrm{in}$. It is important to note that the assumed value of altitude reflects the standpoint of the benchmark above sea level and not the corresponding elevation of the stations as listed in Table 1. Afterwards, the user is required to input the volume of earthwork in BCY (bank cubic yards) in order to determine the productivity rates in LCY/hr (loose cubic yards per hour) as shown in Figure 7. Once completed, the system displays an output report pertaining to the numerical analysis of the earthmoving operations where productivity rates of corresponding equipment for earthmoving operations are tabulated as shown in Figure 8.

As shown in Figure 8, equipment productivity rates are tabulated in LCY/hr where the user may specify the amount of equipment needed to complete the operations along with their corresponding productivity rates as shown in Figure 9. Once completed, the selected fleet along with corresponding productivity rate is automatically extracted and imported into the earthmoving linear scheduling system as shown in Figure 10.

As shown in Figure 10, the daily output of earthmoving operations is determined based on the volume of earthwork involved and the most likely duration required to complete the work. Once completed, the user is required to input optimistic and pessimistic operations durations in order to calculate the corresponding expected durations. Once determined, the proposed system displays a distribution curve that presents the quality of fit of the productivity rates. In the event where the distribution is satisfactory, the user may proceed with the simulated annealing process; otherwise, the user may adjust corresponding productivity rates accordingly. Then, the user is guided towards the simulated annealing process, where initial duration, error difference, and decreasing rate values are required. An initially high project duration value of " 100 " days is inputted in order to escape local minima and reach a near optimum schedule duration. Furthermore, an error difference value of " 0.001 " is specified in order to set the rule of accepting the convergent solution. A decreasing rate of 0.999 is inputted in order to gradually decrease the initial duration and be as close to the ideal annealing process. The user then clicks on the start annealing button in order to determine the minimized earthmoving operation duration as shown in Figure 11. Once completed, the user will click on the global minimization button in order to minimize the total project duration by minimizing the duration of the earthmoving operation that is preceding or succeeding the operation with the global minimum duration as illustrated in Figure 12.

As shown in Figure 12, the proposed system looks for global minima among the expected duration versus earthmoving operation curve. In this study, a polynomial function of the sixth degree is found to be the best fit based on a regression analysis of earthmoving operations and corresponding expected durations. Afterwards, a near optimum line of balance is generated along with the option to adjust earthmoving operations start duration in order to detect potential scheduling conflicts at the conceptual design stage and to modify corresponding consecutive earthmoving operations time buffers as shown in Figure 13.

As illustrated in Figure 13, the expert system presented herein is capable of detecting schedule conflicts at the conceptual design stage by deploying the metaheuristic simulated approach in order to detect scheduling conflicts at the conceptual design stage during the reforming of earthmoving operations sequential combinations. It is important to note that the daily output (LCY) of earthmoving equipment is obtained based on the actual volume of earthwork and the earthmoving operations durations. The user will then adjust the time buffers between earthmoving operations in an iterative process in order to determine the near optimum linear schedule for the total project duration. Typically, fleet productivity rates are not fixed and are subject to daily and possibly hourly fluctuations due to unanticipated occurrences. Hence, it is uncommon to represent a line of balance as a straight line; however, in many cases, engineers and schedulers prefer such representation for indicative purposes at the conceptual design stages. This implies that a productivity over a short duration might be considerably below that which is anticipated and at other times will far exceed the expected production rate. Table 2 summarizes earthmoving operations total durations as obtained from the proposed earthmoving linear schedule system.

It is important to note that the size and composition of the equipment fleet recommended by the expert system differed from those utilized in the case project. The variability in size of equipment fleet is due to scarcity of project site information at the conceptual design stage and availability of equipment at the operational stages. Besides that, the expert system is developed in such a way that a selected equipment fleet is not shared among the earthmoving operations where one type of equipment is selected to perform a single earthmoving operation. Hence, the recommended equipment fleet proposed by the expert system is based on the individual evaluation of equipment productivity rates. Hence, the equipment fleet proposed by the expert system possesses enhanced productivity rates, which contributes towards the size and composition of equipment fleet for a specific project duration. Furthermore, the selection of equipment fleet is directly proportional to daily productivity rates; and, therefore, in order to avoid impractical size of equipment fleet, a comprehensive list of equipment types for each earthmoving operation is organized into seven major categories as discussed earlier. Towards the end, the estimated project durations for diverse soil type and volume are compared to the actual project durations and found to 


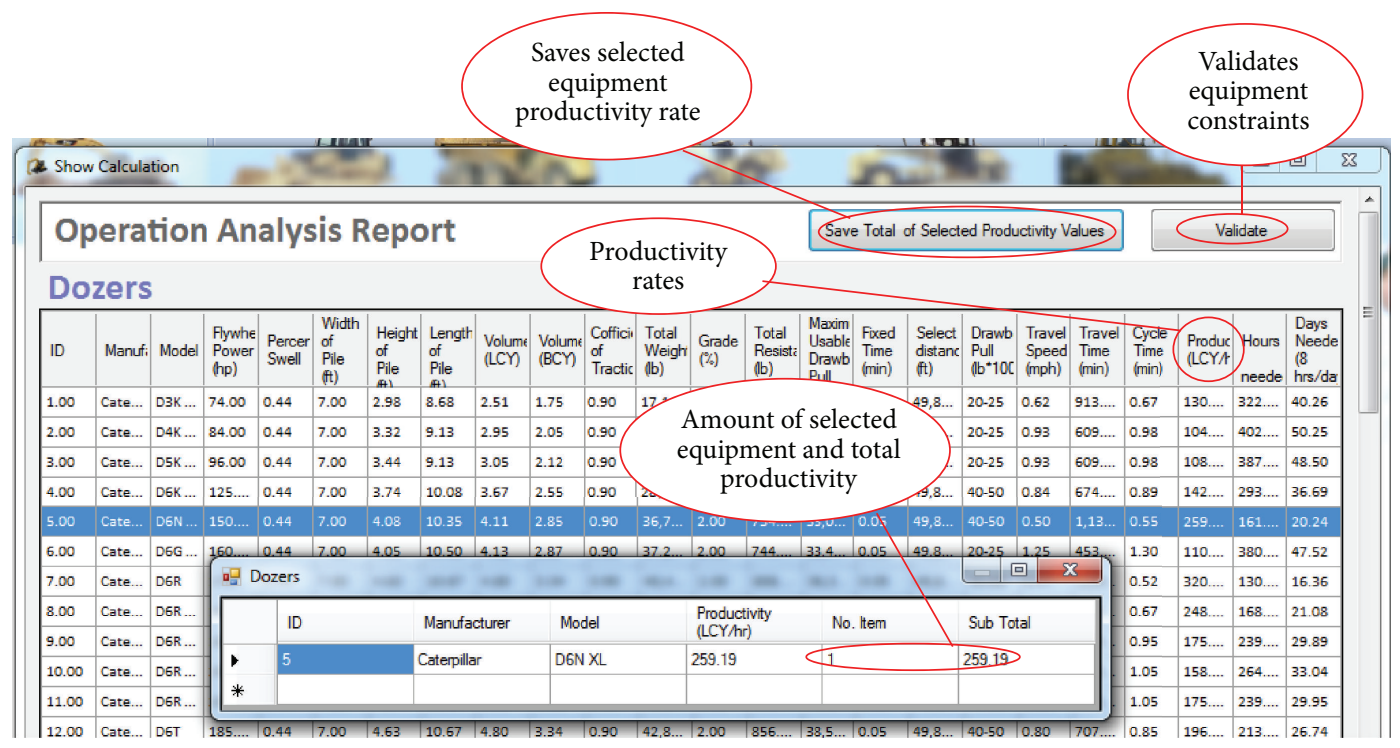

FIGURE 8: Selected earthmoving equipment output report.

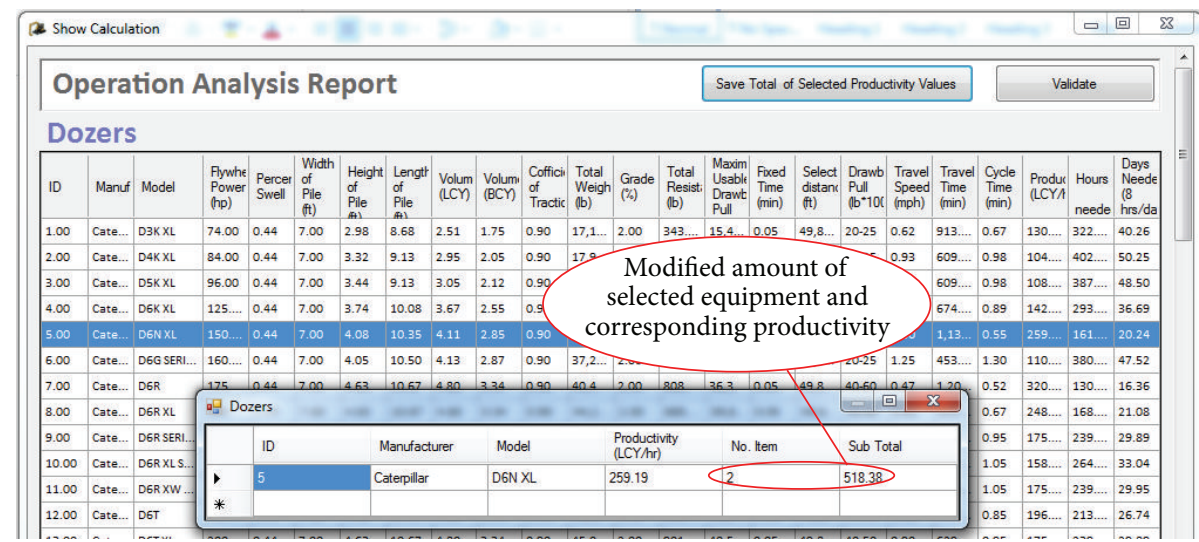

FIGURE 9: Modified amount of selected equipment and corresponding productivity.

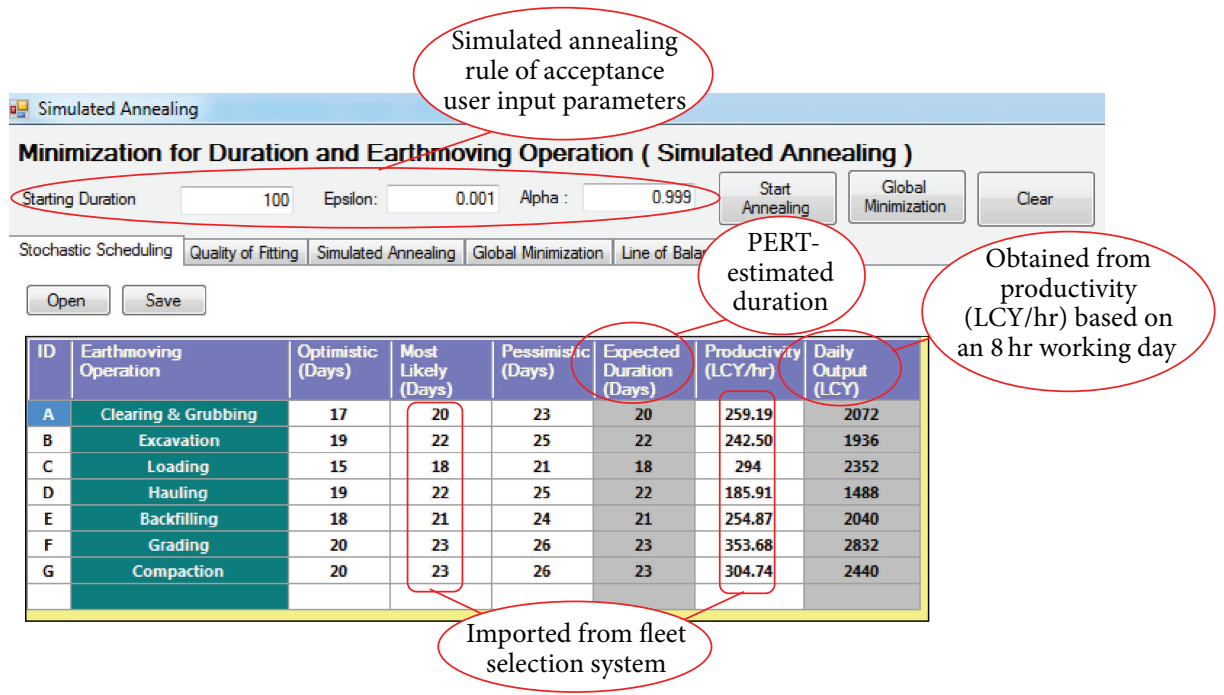

Figure 10: Linear schedule input form I. 
TABLE 2: Comparison of total project duration (days) for diverse soil material and volume.

\begin{tabular}{lccccc}
\hline Soil type & Volume (LCY) & $\begin{array}{c}\text { Actual } \\
\text { Total duration } \\
\text { (days) }\end{array}$ & $\begin{array}{c}\text { Amount of } \\
\text { equipment }\end{array}$ & $\begin{array}{c}\text { Total duration } \\
\text { (days) }\end{array}$ & $\begin{array}{c}\text { Percentage } \\
\text { Amount of } \\
\text { equipment }\end{array}$ \\
\hline Moraine & 41,975 & 67 & $(6,1,1,1)$ & 46 & $(3,1,1,1)$ \\
Granular & 16,250 & 38 & $(6,1,1,1)$ & 32 & $(3,1,1,1)$ \\
Rock & 274,452 & 188 & $(8,1,1,1)$ & 137 & $(4,1,1,1)$ \\
\hline
\end{tabular}

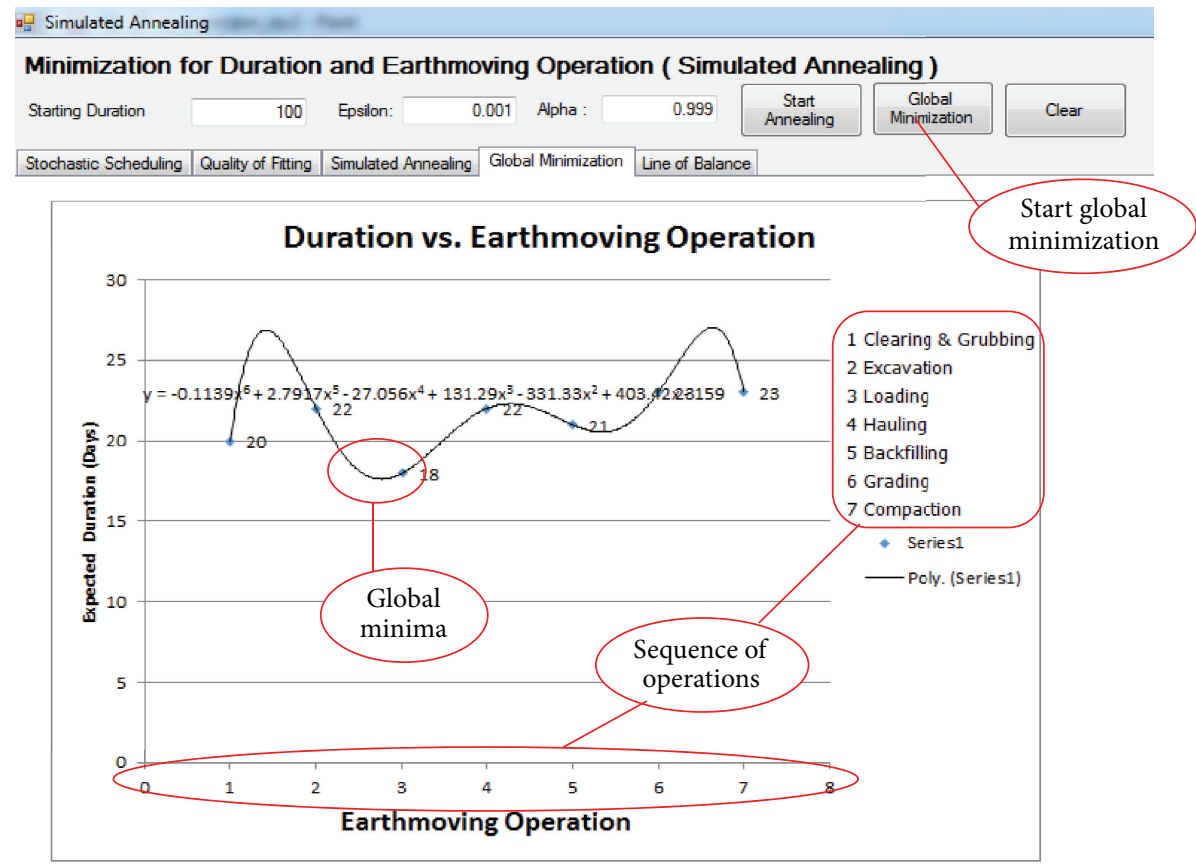

FIGURE 11: Global time minimization form.

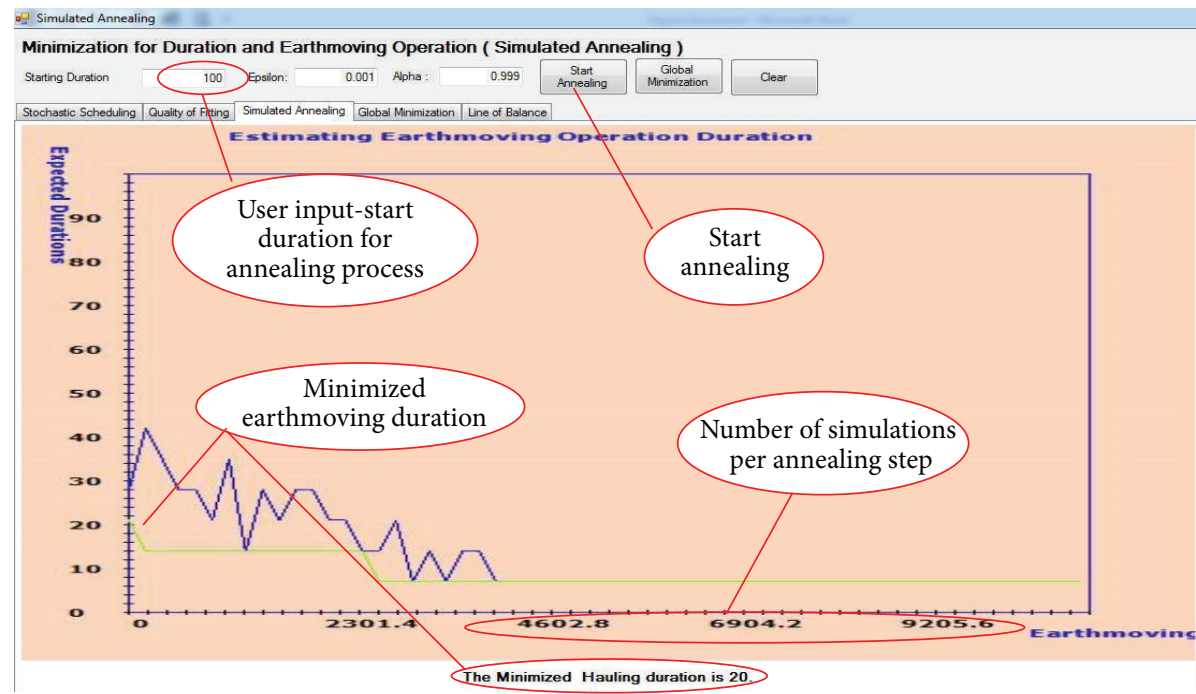

FIGURE 12: Simulated annealing form. 


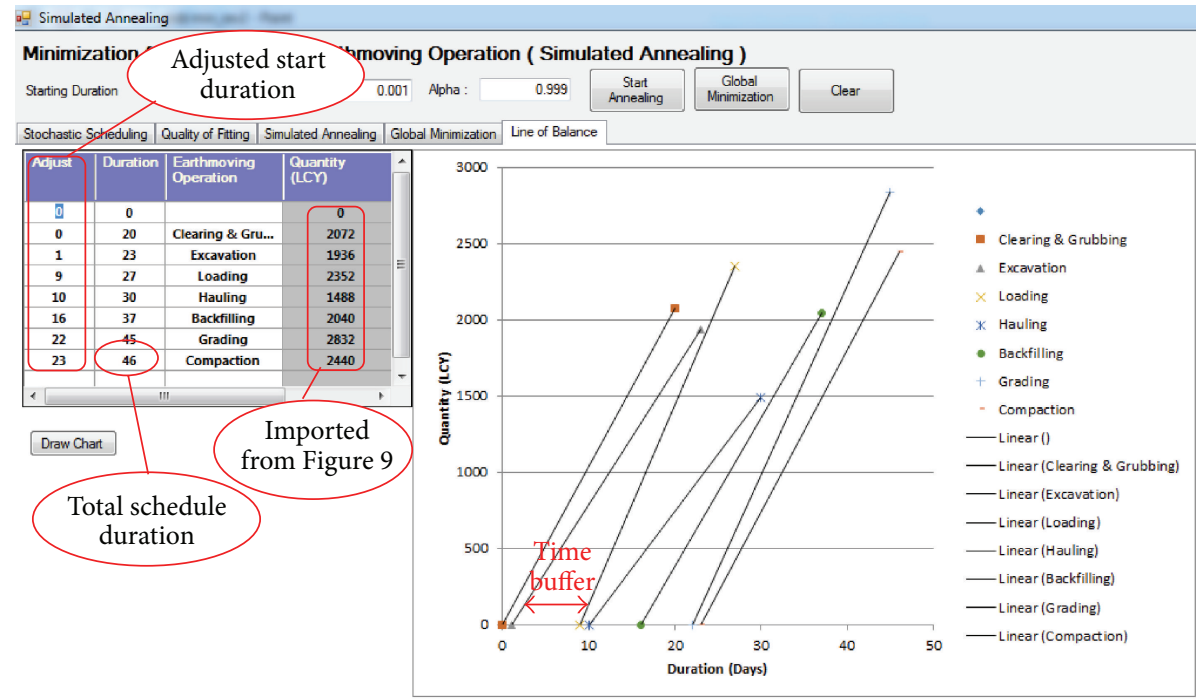

FIgURE 13: Near optimum linear schedule form.

be with a percentage difference ranging between $16 \%$ and $31 \%$ approximately as illustrated in Table 2 . The variation in project duration is due to the difference in the size and composition of the equipment fleet. The range of variation in project duration is somehow acceptable at the conceptual design stage since the overall information pertained to the project is neither fully defined nor detailed.

\section{Summary and Conclusions}

In summary, the proposed expert system is developed to assist engineers and schedulers plan for heavy earthmoving operations. The operation analysis of different types of equipment is undertaken to support equipment selection and develop linear schedules for diverse earthmoving operations. The system is then validated through a case project selected from the literature and its outputs are compared with the actual data. It is apparent that the types of soil and user entries highly influence the accuracy of the proposed system. Also, it is concluded that the system possesses some limitations with regard to rock material. That, in fact, requires much more complex studies to be conducted in order to select the required fleet for this type of work, such as earthwork involving mining construction. It is necessary to mention that the proposed integrated system is developed as an estimation tool that can be used to estimate the required time for a particular earthmoving project. Estimation results are based on user entries and are homogenous in type. The homogeneity of near optimum results is one major limitation of the proposed system as it provides equipment productivity rates that are capable of undertaking the work for diverse soil properties except for rock material. This is an ongoing research and its authors are working on strengthening the system by enhancing the decision support tool and by incorporating additional optimization parameters and constraints to it. This will be done by considering more equipment specifications data and equipment alternatives.
For instance, this system is limited to one major heavy equipment manufacturer, Caterpillar, where other types of equipment manufacturers may be included. Furthermore, more specific factors that can be applied for specific types of equipment (i.e., scrapers) will be considered. In general, it is not possible for the proposed system to predict fleet productivity accurately and without errors. This is simply due to the nature and characteristics of construction projects. The contribution of this study mainly resides in the development of a linear scheduling method for capturing the variability in equipment productivity rates. Furthermore, the deployment of a simulated annealing approach is a novel approach for scheduling earthmoving operations. It is also important to note that the proposed global minimization approach will significantly enhance overall project duration and result in time and cost savings in general and specifically for combinational type of earthmoving operations such as loadinghauling, compacting-grading, and backfilling-compacting. This paper is the first attempt to incorporate equipment operation analysis as the framework of the linear scheduling process. The main advantage of the proposed system is the ability to automate user input data interface with equipment operation analysis. The proposed system can be utilized even in projects that involve a large volume of earthwork. This capability gives the system great advantages over other time minimization algorithms, prototypes, or models published and presented in the literature.

\section{Competing Interests}

The authors declare that they have no competing interests.

\section{References}

[1] K. Agrawal, A. Benoit, L. Magnan, and Y. Robert, "Scheduling algorithms for linear workflow optimization," in Proceedings of the 24th IEEE International Symposium on Parallel \& Distributed 
Processing (IPDPS '10), pp. 1-12, IEEE, Atlanta, Ga, USA, April 2010.

[2] L. Song and S. H. Lee, "Stochastic look-ahead scheduling method for linear construction projects," Journal of Risk Analysis and Crisis Response, vol. 2, no. 4, pp. 252-260, 2012.

[3] M. König and U. Beißert, "Construction scheduling optimization by simulated annealing," in Proceedings of the 26th International Symposium on Automation and Robotics in Construction (ISARC '09), 2009.

[4] C. Srisuwanrat and P. G. Ioannou, "The investigation of leadtime buffering under uncertainty using simulation and cost optimization," in Proceedings of the 15th Annual Conference of the International Group for Lean Construction (IGLC '15), pp. 580-589, Ann Arbor, Mich, USA, July 2007.

[5] W. Liu, I. Flood, and R. Issa, "Simulation and optimization of linear construction projects," Journal of Computing in Civil Engineering, pp. 1-11, 2005.

[6] S. Nunnally, Managing Construction Equipment, Prentice-Hall, Englewood Cliffs, NJ, USA, 1977.

[7] J. Schaufelberger, Construction Equipment Management, Prentice-Hall, Upper Saddle River, NJ, USA, 1999.

[8] A. Jrade, N. Markiz, and N. Albelwi, "An economical operation analysis optimization model for heavy equipment selection," International Journal of Civil and Environmental Engineering, vol. 6, pp. 40-45, 2012.

[9] A. Jrade and N. Markiz, "A decision-support model utilizing a linear cost optimization approach for heavy equipment selection," in Proceedings of the Construction Research Congress, pp. 100-109, ASCE, West Lafayette, Ind, USA, May 2012.

[10] N. Markiz and A. Jrade, "Integrating bridge information modeling (BrIM) with cost estimation at the conceptual design phase of bridges," in Proceedings of the 4th Construction Specialty Conference (CSCE '13), pp. 1-10, Montreal, Canada, 2013.

[11] W. Shang and J. Fortes, "Time optimal linear schedules for algorithms with uniform depdencies," in Proceedings of the International Conference on Systolic Arrays, pp. 393-402, IEEE, 1988.

[12] J. Tobochnik and H. Gould, "Teaching statistical physics by thinking about models and algorithms," American Journal of Physics, vol. 76, no. 4-5, pp. 353-359, 2008.

[13] U. Beißert, M. König, and J. Bargstädt, "Constraint-based simulation of outfitting processes in building engineering," in Proceedings of the 24th Conseil International du Bâtiment W78 Conference, pp. 183-190, 2007.

[14] J. Dreo, P. Siarry, A. Petrowski, and E. Taillard, "Metaheuristics for hard optimization," Journal of Mathematical Methods of Operations Research, vol. 66, no. 3, pp. 557-558, 2007.

[15] S. Ledesma, G. Avina, and R. Sanchez, "Practical considerations for simulated annealing implementation," in Simulated Annealing, pp. 401-420, InTech, Vienna, Austria, 2008.

[16] M. Marzouk, Optimizing earthmoving operations using computer simulation [Ph.D. thesis], University of Concordia, Montreal, Canada, 2002. 


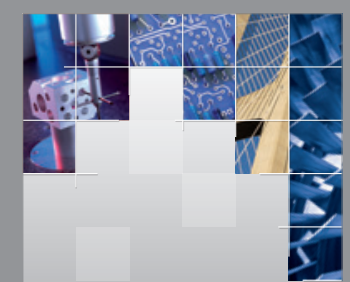

\section{Enfincering}
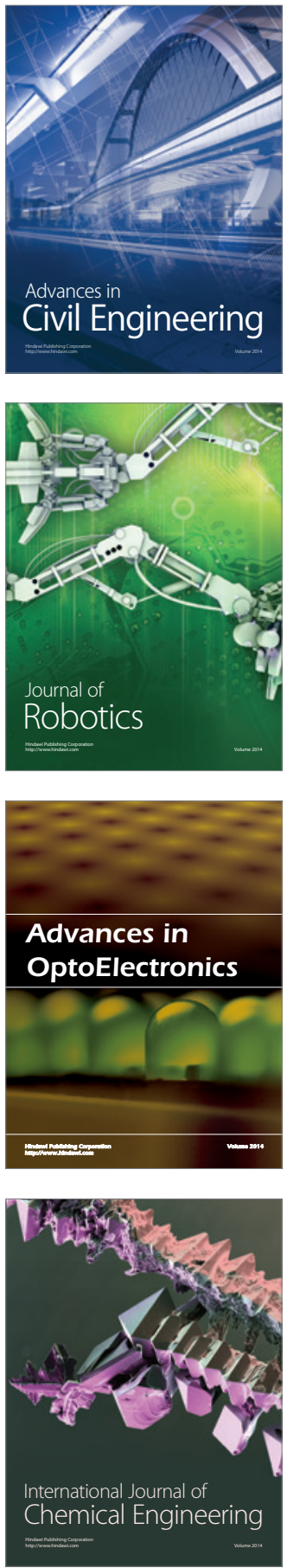

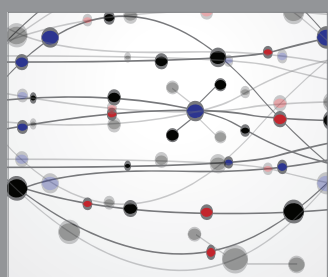

The Scientific World Journal

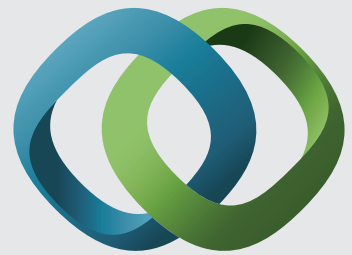

\section{Hindawi}

Submit your manuscripts at

http://www.hindawi.com
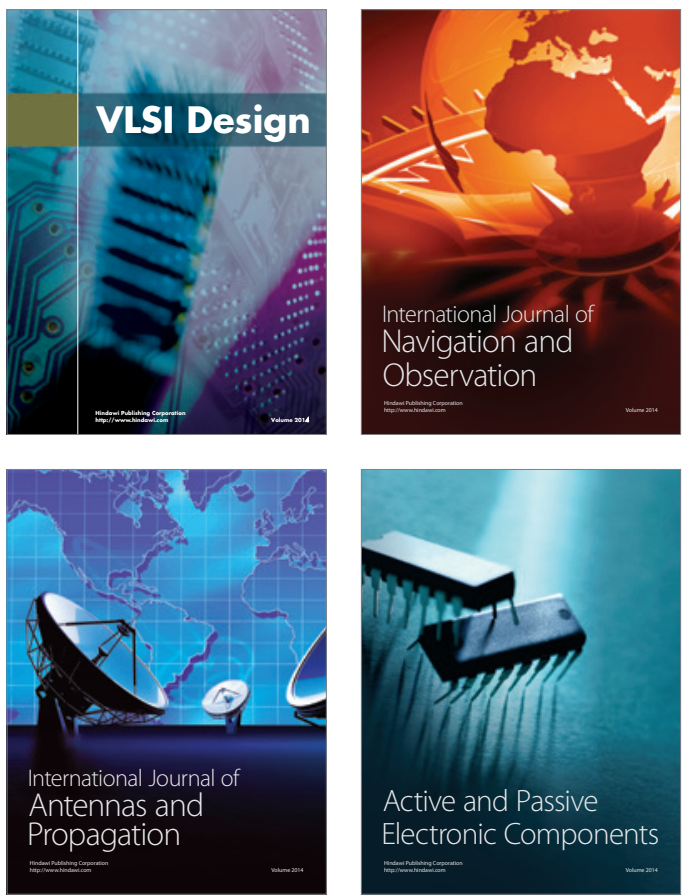
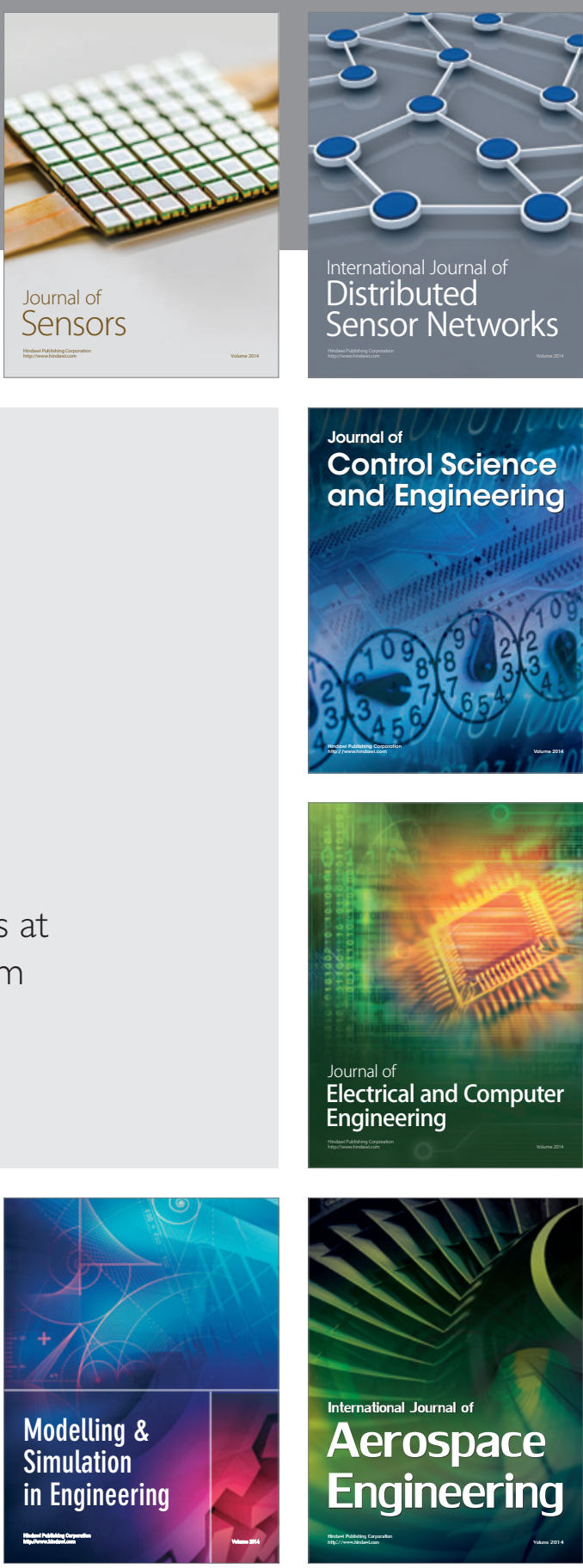

International Journal of

Distributed

Sensor Networks

Journal of

Control Science

and Engineering
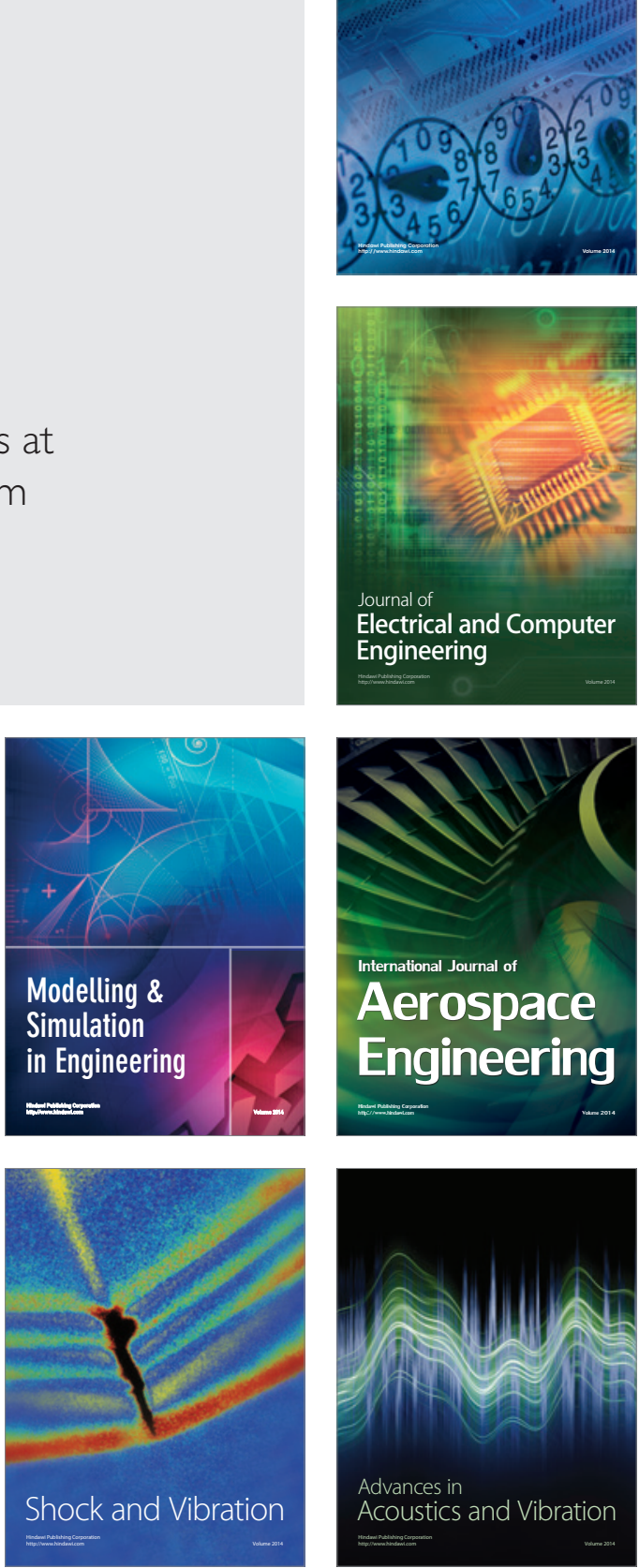